Pacific Journal of Mathematic 


\section{PROJECTIVE INJECTIVE MODULES}

\section{J. P. JANS}

1. Introduction. In this paper we prove several theorems about rings having a generous supply of projective injective modules. This is a curious class of rings. For instance, every module over a semisimple ring with minimum condition is both projective and injective, while over the integers only the zero module has this property. On the other hand, for some non-semisimple rings, Quasi Frobenius rings [5], every projective module is injective. For others no non-trivial projective module is injective (for example, a primary algebra over a field with radical square zero and having vector space dimension greater than two).

We begin our study in $\S 2$ by considering primitive rings. We give (Theorem 2.1) a necessary and sufficient condition for a primitive ring to have a faithful projective injective irreducible module. By means of this condition we prove a structure theorem (Corollary 2.3) for rings having both a left and a right injective projective irreducible module with the same anihilator.

In $\S 3$ we generalize both halves of a theorem originally proved by Thrall for finite dimensional algebras [10, Theorem 5]. This theorem states that a necessary and sufficient condition for the minimal injective [3] of the ring to be projective is that the ring have a faithful injective module which is a direct summand of every faithful module. We prove this theorem in one direction for semi-primary rings and, in the other direction, for rings with the ascending chain condition. It should be noted that we have rephrased the theorem to eliminate the duality given by the field. We find that this can be replaced by the dual concepts, projective and injective.

Throughout the paper we shall only consider rings with identity 1 and modules over such rings on which 1 acts like identity. "Minimum condition" means minimum condition on left ideas [1].

The author wishes to express his appreciation to John Walter for many stimulating conversations which contributed to the formulation of this paper. We also wish to thank Alex Rosenberg for suggesting clear concise proofs of Theorems 2.1 and 3.2.

2. Projective injective irreducibles. We shall begin by considering primitive rings. Recall that a (right) primitive ring $R$ has a faithful irreducible right module $M[7, \mathrm{p}$. 4]. The module $M$ is always the homomorphic image of $R$, and if $M$ is projective then $M$ is induced by

Received Jaunary 28, 1959. The author was supported by the National Science Foundation. 
a minimal right ideal of $R$. That is, $R$ is a primitive ring with minimal right ideals. Conversely, if $R$ is a primitive ring with minimal right ideals then the faithful irreducible module is induced by an idempotent generated (= direct summand) right ideal of $R$. Thus, the faithful irreducible is projective.

In the following we shall study primitive rings with minimal right ideals and we shall establish a necessary and sufficient condition for the faithful irreducible module of such a ring $R$ to be injective. We are greatly aided in this study by the rich structure theory for these rings; see for example Jacobson's book [7, Chapter IV].

Using the notation and the structure theorem from [7, p. 75], we have $S=F(M, N) \subset R \subset L(M, N)$ where $M, N$ are dual spaces over a division ring $D$ and $M(N)$ is a right (left) irreducible faithful projective $R$-module. $S$ is the socle of $R$.

Theorem 2.1. The module $M$ is $R$ injective if and only if $M=$ $N^{*}=\operatorname{Hom}_{D}(N, D)$.

Proof. If $M=\operatorname{Hom}_{D}(N, D)$ then by Prop. 1.4 p. 107 of [2], $M$ is $R$ injective.

For the converse, assume that $M$ is $R$ injective. In this case, it is enough to show that for every maximal right ideal $J$ of $S$ there is a nonzero element $a$ of $S$ such that $a J=0$. Then the left ideal $S a$ contains an idempotent $e \neq 0$ such that $e J=0$ and $J$ is a modular [7] (called regular in [9]) right ideal. But Rosenberg has shown [9, p. 131] that if every maximal right ideal of $S$ is modular then $M=N^{*}=$ $\operatorname{Hom}_{D}(N, D)$.

Identify $M$ with a minimal right ideal of $S$. Since $J$ is maximal in $S$ we can consider the $R$ exact sequence of modules

$$
O \longrightarrow J \longrightarrow S \stackrel{\theta}{\longrightarrow} M \longrightarrow O \text {. }
$$

Since $M$ is $R$ injective by [2, Th 3.1, p. 8] the homomorphism $\theta$ has the form $\theta(s)=$ as for some $a \neq 0$ in the right ideal $M$ of $S$. But since $\operatorname{Ker} \theta=J, a J=0$. Theorem 2.1 then follows from the remarks above.

One should note that the corresponding theorem with right and left interchanged is proved analogously, hence we have the following

COROLlaRY 2.2. If $R$ is a primitive ring then $R$ is a simple ring with minimum condition if and only if $R$ has both a left and a right faithful irreducible projective injective module.

Proof. If $R$ is a simple ring with minimum condition then it has faithful irreducible left and right modules [7, p. 39] and every module 
over such a ring is both projective and injective [2, p. 11].

To show the converse, we appeal to the theorem. Using the notation of the theorem, $M=N^{*}$ and $M^{*}=N$. But we know [7, p. 68], that this can only happen when both have finite dimension over $D$. In this case $R$ is isomorphic to all transformations on $M$ and is a simple ring with minimum condition [7, p. 39].

The theorem and its corollary also have applications to any ring having left and right projective injective irreducibles. It is clear that if a ring $R$ can be written as a ring direct sum $S+K$ where $S$ is a simple ring with minimum condition, then $R$ has both a left and a right projective injective irreducible module, each having anihilator $K$. It is interesting to note that the converse is also true.

CoRollary 2.3. If $R$ has both a left and a right projective injective irreducible, each having anihilator $K$, then $R=S+K$ (ring direct sum) where $S$ is a simple ring with minimum condition.

Proof. Under the above assumptions $R / K$ is both a left and a right primitive ring and the faithful irreducible left and right modules considered as $R / K$ modules are still projective and injective. Thus, by Corollary $2.2, R / K$ is a simple ring with minimum condition and both as an $R$ module and as an $R / K$ module is the direct sum of a finite number of copies of the left irreducible projective injective module. Thus the sequence of left $R$ modules $0 \rightarrow K \rightarrow R \rightarrow R / K \rightarrow 0$ splits and $R=S \oplus K$, left $R$ direct. The proof will be established if we can show that $S$ is really an ideal of $R$.

Certainly, $K S=(0)$ because $S$ is the direct sum of modules anihilated by $K$. Let $k$ belong to $K$ and consider the left ideal $S k$ contained in $K$. It is clear that $(S k)^{2}=S k S k=(0)$ because $k$ anihilates $S$ on the left. Suppose that $S k$ is not zero. In this case, $S k$ is the homomorphic image of the completely reducible module $S$ and is the direct sum of a finite number of injective irreducible modules. But that makes $S k$ injective and a direct summand of $R$. However, this contradicts the fact that $S k$ is square zero, since direct summands of $R$ are idempotent generated. Thus we have established that $S k=(0)$ and that the decomposition given above is a ring direct sum.

REMARK. There is a one-sided version of Corollary 2.3, in which one assumes only the existence of a projective injective irreducible left module plus the ascending chain condition on left ideals in $R$ modulo its Jacobson radical. The conclusion is the same. However, the conclusion is two sided, so the existence of a projective injective left irreducible and the above mentioned chain condition (or semi-primary, etc.) implies the existence of a projective injective right irreducible. 
3. Minimal faithfuls and minimal injectives. Following Thrall's paper [10], we shall say that the ring $R$ has a minimal faithful left module $M$ if $M$ is a faithful injective module and if $M$ appears as a direct summand of every faithful module. It is clear that $M$ must be projective, for the ring itself is a faithful projective module. $M$ will always be isomorphic to some left ideal direct summand of $R$.

If $T$ is any $R$ module, the minimal injective $Q(T)$ of $T$ is the unique "smallest" injective module containing $T$ as a submodule, [3]. Using these two concepts, we can prove a generalization of one half of a theorem of Thrall [10, Theorem 5]. Thrall proved it for finite dimensional algebras over a field.

THEOREM 3.1. If $R$ is right Noetherian and if $R$ has a minimal faithful left module $M$ then $Q(R)$, the left minimal injective of $R$, is projective.

Proof. As noted above $M$ must be isomorphic to a projective injective left ideal which we also denote by $M$. In $R$ consider the collection of right ideals generated by finite sets of elements of $M$. Since we have assumed $R$ to be right Noetherian, there is in this collection a maximal right ideal $H$ generate by $x_{1}, \cdots, x_{n}$ belonging to $M$. Since $H$ is maximal with respect to this property, we know that $M \subset H$. For if not, $H$ could be enlarged by adjoining another generator from $M$.

If $x$ is in $R$ and $x x_{i}=0$ for $i=1, \cdots, n$, then $x H=(0)$ and consequently $x M=(0)$. But $M$ is faithful, so $x=0$. Now let $Q$ be the direct sum of $n$ copies of $M$ and for $x$ in $R$ define $\theta: R \rightarrow Q$ by letting the $i$ th component of $\theta(x)$ be $x x_{i}$. This is a left module homomorphism of $R$ into $Q$ and, by the remark above, is a monomorphism. $Q$ is projective and injective since it is the direct sum of a finite number of projective injective modules. The minimal injective of $R$ is a direct summand of $Q$ and is therefore projective.

We should note that if $R$ is both left and right Noetherian and has a minimal faithful left module then the minimal injective of any projective module is projective. This follows from the fact that every free module can be embedded in a projective injective module, a direct sum of copies of $M$. We need the assumption that $R$ is left Noetherian to insure that the direct sum of left injectives is injective. Compare this to the definition of Quasi Frobenius ring [5]: "Every projective is injective".

To prove the other half of Thrall's theorem we consider the class of semi-primary rings. The ring $R$ is said to be semi-primary if it has a nilpotent Jacobson radical $N$ and $R / N$ has minimum condition on left ideals. An important property of semi-primary rings is the fact that 
every module over such a ring has minimal submodules. For, if $M$ is a module over the semi-primary ring $R$ with radical $N$ then in the sequence $M \supset N M \supset \cdots \supset N^{r} M=(0)$ of submodules of $M$ there is a point where $N^{k} M \neq(0)$ but $N^{k+1} M=(0) . \quad N^{k} M$, a module over $R / N$, is the direct sum of irreducibles each of which is minimal. Note also that $R$ has only a finite number of nonisomorphic irreducible modules.

THEOREM 3.2. If $R$ is a semi-primary ring and if the left minimal injective $Q(R)$ of $R$ is projective then $R$ has a minimal faithful module.

Proof. By the remark above, we know that $R$ itself has minimal left ideals. Let $M_{1}, \cdots, M_{n}$ be one each of the non-isomorphic minimal left ideals of $R$. From [8], we know that the minimal injective $Q\left(M_{i}\right)$ of $M_{i}$ is indecomposable. In addition each $Q\left(M_{i}\right)$ is projective since it appears as a direct summand of $Q(R)$. But the projective indecomposable modules over a semi-primary ring actually appear as left ideal direct summands of the ring [4, p. 331]. Thus each $Q\left(M_{i}\right)$ is isomorphic to a projective injective indecomposable left ideal $L_{i}$ of $R$. Note that for $i \neq j, L_{i}$ is not isomorphic to $L_{j}$ since each has a unique minimal submodule [8] and these are not isomorphic.

Let $M$ be the direct sum of the modules $L_{i}$, we wish to show that $M$ is the minimal faithful module for $R$. From its definition it is projective and injective. If $M_{\alpha}$ is a minimal ideal of $R, M_{\alpha}$ is isomorphic to a minimal submodule of $M$. Since $M$ is injective that isomorphism has the form $x \rightarrow x m$ for some $m$ in $M\left[2, \mathrm{p}\right.$. 8]. Hence $M_{\alpha}$ does not anihilate $M$. If no minimal left ideal of $R$ anihilates $M$, then no nonzero left ideal anihilates $M$ and $M$ is faithful.

Now let $T$ be an $R$ module such that $M_{i} T \neq 0$. Then there exists $t$ in $T$ such that $M_{i} t \neq 0$. Consider the homomorphism $\Sigma(x)=x t$ of $L_{i}$ into $T$. This homomorphism restricted to $M_{i}$ is not zero and since $M_{i}$ is the unique minimal submodule of $L_{i}, \Sigma$ is actually a monomorphism of $L_{i}$ into $T . \quad L_{i}$ is injective so $T=L_{i} \oplus T_{1}$.

From the preceding argument we conclude that for $i \neq j M_{i} L_{j}=0$ since $L_{i}$ and $L_{j}$ are indecomposable and not isomorphic. Now let $F$ be a faithful $R$ module. Since $M_{1} F \neq 0$, the argument above shows that $F=L_{1} \oplus F_{1}$ where $M_{i} F_{1} \neq 0$ for $i>1$. Continuing inductively, $F_{i-1}=$ $L_{i} \oplus F_{i}$ where $M_{j} F_{i} \neq 0$ for all $j>i$. Thus we see $F=M \oplus F_{n}$ and $M$ appears as a direct summand of every faithful $R$ module. This completes the proof of Theorem 3.2.

REMARK. Since a ring with minimum condition is both semi-primary and Noetherian, both halves of Thrall's theorem hold for these rings. 


\section{REFERENCES}

1. Artin, Nesbitt and Thrall, Rings with Minimum Condition, Ann Arbor (1944).

2. Cartan and Eilenberg, Homological Algebra, Princetion (1956).

3. Eckmann and Schopf, Uber Injective Moduln, Arch. Math. 4 (1953).

4. S. Eilenberg, Homological dimension and syzygies, Ann. of Math. 64 (1956), 328.

5. Eilenberg, Nagao and Nakayama, On the dimensions of modules and algebras II Nagoya Math. Journal 9 (1955), 1-16.

6. N. Jacobson, Lectures in Abstract Algebra I, Van Nostrand: 1951.

7. - Structure of rings, Amer. Math. Soc. Coll. Publication XXXVII, 1956.

8. E. Matlis, On injective modules, Pacific J. Math. 8 (1958), 511-528.

9. A. Rosenberg, Subrings of simple rings with minimal ideals, Trans. Amer. Math. Soc. 73 (1952), 115-138.

10. R. M. Thrall, Some generalizations of quasi Frobenius algebras, Trans. Amer. Math. Soc. 64 (1948) 173-183.

UNIVERSITY OF WASHINGTON 


\section{PACIFIC JOURNAL OF MATHEMATICS}

EDITORS

David Gilbarg

Stanford University

Stanford, California

R. A. Beaumont

University of Washington

Seattle 5 , Washington
A. L. Whiteman

University of Southern California

Los Angeles 7, California

L. J. PAIGE

University of California

Los Angeles 24, California

\section{ASSOCIATE EDITORS}

\author{
E. F. BECKENBACH \\ C. E. BURGESS \\ E. HEWITT \\ A. HORN
}

\author{
V. GANAPATHY IYER \\ R. D. JAMES \\ M. S. KNEBELMAN \\ L. NACHBIN
}
I. NIVEN
E. G. STRAUS
T. G. OSTROM
G. SZEKERES
H. L. ROYDEN
F. WOLF
M. M. SCHIFFER
K. YOSIDA

\section{SUPPORTING INSTITUTIONS}

\author{
UNIVERSITY OF BRITISH COLUMBIA \\ CALIFORNIA INSTITUTE OF TECHNOLOGY \\ UNIVERSITY OF CALIFORNIA \\ MONTANA STATE UNIVERSITY \\ UNIVERSITY OF NEVADA \\ OREGON STATE COLLEGE \\ UNIVERSITY OF OREGON \\ OSAKA UNIVERSITY \\ UNIVERSITY OF SOUTHERN CALIFORNIA
}

\author{
STANFORD UNIVERSITY \\ UNIVERSITY OF TOKYO \\ UNIVERSITY OF UTAH \\ WASHINGTON STATE COLLEGE \\ UNIVERSITY OF WASHINGTON \\ $* \quad * \quad *$ \\ AMERICAN MATHEMATICAL SOCIETY \\ CALIFORNIA RESEARCH CORPORATION \\ HUGHES AIRCRAFT COMPANY \\ SPACE TECHNOLOGY LABORATORIES
}

Mathematical papers intended for publication in the Pacific Journal of Mathematics should be typewritten (double spaced), and the author should keep a complete copy. Manuscripts may be sent to any one of the four editors. All other communications to the editors should be addressed to the managing editor, L. J. Paige at the University of California, Los Angeles 24, California.

50 reprints per author of each article are furnished free of charge; additional copies may be obtained at cost in multiples of 50 .

The Pacific Journal of Mathematics is published quarterly, in March, June, September, and December. The price per volume (4 numbers) is $\$ 12.00$; single issues, $\$ 3.50$. Back numbers are available. Special price to individual faculty members of supporting institutions and to individual members of the American Mathematical Society: $\$ 4.00$ per volume; single issues, $\$ 1.25$.

Subscriptions, orders for back numbers, and changes of address should be sent to Pacific Journal of Mathematics, 2120 Oxford Street, Berkeley 4, California.

Printed at Kokusai Bunken Insatsusha (International Academic Printing Co., Ltd.), No. 6, 2-chome, Fujimi-cho, Chiyoda-ku, Tokyo, Japan.

PUBLISHED BY PACIFIC JOURNAL OF MATHEMATICS, A NON-PROFIT CORPORATION

The Supporting Institutions listed above contribute to the cost of publication of this Journal, but they are not owners or publishers and have no responsibility for its content or policies. 


\section{Pacific Journal of Mathematics}

\section{Vol. 9, No. 4 \\ August, 1959}

Frank Herbert Brownell, III, A note on Kato's uniqueness criterion for

Schrödinger operator self-adjoint extensions ............... 953

Edmond Darrell Cashwell and C. J. Everett, The ring of number-theoretic

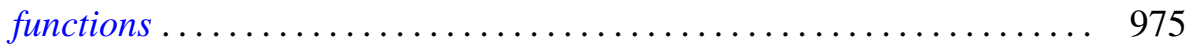

Heinz Otto Cordes, On continuation of boundary values for partial

differential operators ............................. 987

Philip C. Curtis, Jr., n-parameter families and best approximation . . . . . . 1013

Uri Fixman, Problems in spectral operators . . . . . . . . . . . . . . . 1029

I. S. Gál, Uniformizable spaces with a unique structure .............. 1053

John Mitchell Gary, Higher dimensional cyclic elements ............ 1061

Richard P. Gosselin, On Diophantine approximation and trigonometric

polynomials ..................................... 1071

Gilbert Helmberg, Generating sets of elements in compact groups ........ 1083

Daniel R. Hughes and John Griggs Thompson, The H-problem and the

structure of $H$-groups .................................. 1097

James Patrick Jans, Projective injective modules ................. 1103

Samuel Karlin and James L. McGregor, Coincidence properties of birth and

death processes ..................................... 1109

Samuel Karlin and James L. McGregor, Coincidence probabilities ........ 1141

J. L. Kelley, Measures on Boolean algebras ................... 1165

John G. Kemeny, Generalized random variables ................... 1179

Donald G. Malm, Concerning the cohomology ring of a sphere bundle ... . . 1191

Marvin David Marcus and Benjamin Nelson Moyls, Transformations on

tensor product spaces .................................. 1215

Charles Alan McCarthy, The nilpotent part of a spectral operator ........ 1223

Kotaro Oikawa, On a criterion for the weakness of an ideal boundary

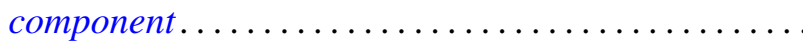

Barrett O'Neill, An algebraic criterion for immersion . . ............... 1239

Murray Harold Protter, Vibration of a nonhomogeneous membrane ... . . . . 1249

Victor Lenard Shapiro, Intrinsic operators in three-space . . . . . . . . . . . 1257

Morgan Ward, Tests for primality based on Sylvester's cyclotomic

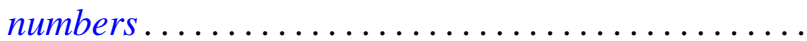

L. E. Ward, A fixed point theorem for chained spaces ....

Alfred B. Willcox, Šilov type $C$ algebras over a connected locally compact

abelian group..................................... 1279

Jacob Feldman, Correction to "Equivalence and perpendicularity of

Gaussian processes" ........................ 